\title{
In the face of COVID-19, SADA continues to deliver to its members
}

SADJ July 2020, Vol. 75 No. 6 p289 - p290

KC Makhubele

CEO of SADA

SADA is proud to be amongst the small elite group of professional associations representing healthcare practitioners, fully recognised worldwide. Recently we held both the SADA Annual General and National Council Meeting virtually through a meetings electronic platform - Zoom.

We have indeed entered a new era in the history of the Association as these were the first members meetings held electronically. We are pleased that members registered and attending were able to actively participate and exercise their voting rights on various issues tabled at these meetings.

All Branch, National Council, Board and Board Committee meetings will be conducted by electronic means in the foreseeable future. It is also reported with regret that the 2020 Dental Congress and Exhibition had to be cancelled at the Emperors Palace Conference Centre in Gauteng. Several alternatives and strategies are being investigated to meet the educational and CPD needs of members for the year.

We are proud to announce the appointment of Dr Rhonin Naidoo (Kwa-Zulu Branch) as the new President of the Association and Dr Anthony Julius (North-West Branch) as the Vice-President of the Association, they will both serve for a two-year term. On behalf of the Board, National Council and management, we welcome them and express our profound and heartfelt congratulations on their appointment. We wish them every success and we are confident they will proudly represent our Association for the next two years.

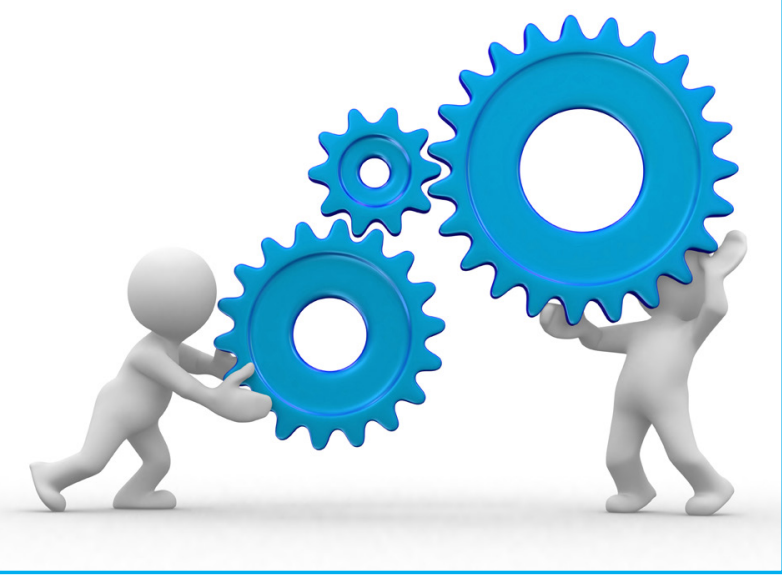

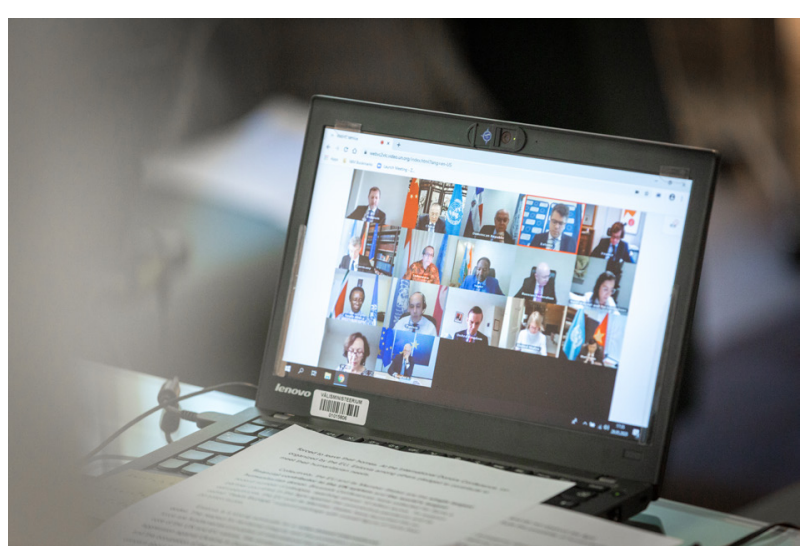

Image Source: Jürgen Randma / CC BY-SA (https://creativecommons.org/ licenses/by-sa/4.0)

As we enter advanced level 3 of the COVID-19 pandemic, the extreme challenges facing our members are becoming more dire with shortages of required PPE, staff testing positive for COVID-19, patients fearful about consulting their dentists, some practitioners contemplating earlier retirement than anticipated, as a measure of supporting our members, we have offered two months free membership. Members have been requested to provide their banking details to permit the Association refund them much needed funds.

The extended Dental Practice Committee together with the different workstreams specifically mandated to deal with different aspect of the pandemic have been hard at work from the start of the lockdown and after and we are proud to make available to the entire dental fraternity Protocols to guide members working under COVID-19 conditions.

The protocols have been enthusiastically received by all stakeholders we have been engaging with not only in South Africa, but even internationally it is seen as a leading protocol document. We are happy to report that dental professional associations abroad are equally impressed with the contents of a well-researched document.

The different workstreams have also been hard at work holding webinars every week keeping members properly informed about the different challenges facing the profession during this time. Many more are being planned and we have consciously decided to make these available to members and non-members alike in the interests of fulfilling our duties as citizens in contributing to the prevention of the spread of the disease. 
The electronic book titled "Infection Control Guidelines for Oral Health Care" by Dr Jeanné Oosthuysen and co-authors is available for members on the website which we hope guide our members in keeping their patients, staff and themselves safe.

Members were introduced to services of two labour consultants who will offer their services on labour related challenges members are likely to face now and in the coming months. Members are encouraged to utilise their services to prevent unnecessary and costly labour disputes. Several guidelines have been issued for the benefit of practitioners as employers.

Engagements with medical schemes, medical scheme administrators and third-party funders are ongoing as regards providing benefits for additional PPE for AGPs and non-AGPs performed by practitioners.

While some schemes are willing to consider benefits for the practitioner and assistants, others have resisted despite the fact these are also required for the protection of their members.

Members have also started the debate about the continued reliance of the profession on third party funders and its devastating impact on the future of the profession. To this end, the Board has grappled with this subject and will survey members about their practice circumstances and reliance in these tough economic times.
Perhaps the time has come for the profession to reconsider their position now and in the post pandemic era, to make their patients to whom they provide treatment responsible to them for payment of services rendered. It is now time that the profession takes charge of their own practices and like other businesses, rely on their customer to pay for services received.

Members would also have followed through webinars that Dental Protection is seeking to establish service centres and more personnel to assist members at a local level to provide more efficient and quicker turnaround for members seeking guidance, facing complaints and/or claims.

We are also pleased to announce the appointment of the erstwhile President, Professor P Moipolai to the South African Dental Technicians Council as a member attached to an academic institution. The Chairperson of the SADA Board of Directors, Dr R Putter was successfully appointed to the PPS Trust Board. We wish them both success in their appointment. The Board also appoint two new independents, Mrs Carina Wessels (Admitted Advocate of the High Court of South Africa and Executive: Governance, Legal and Compliance at Alexander Forbes) and Mr Hiten Keshave (A charted accountant and CFO at PRP Solutions (Pty) Ltd.

Amidst all of the challenges the profession is facing, the business of the association continues.

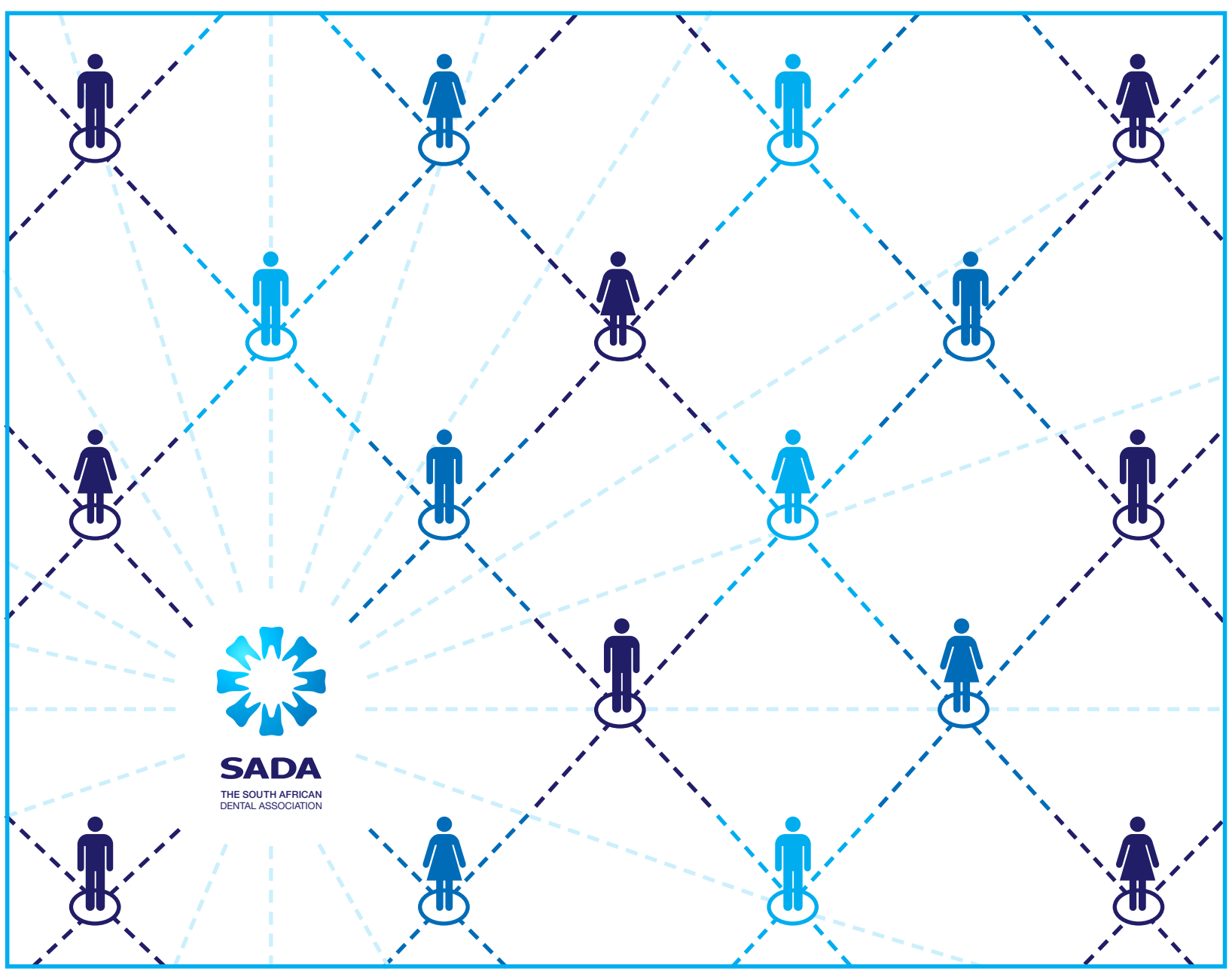

